\title{
The Association of Radiation Dose-Fractionation and Immunotherapy Use With Overall Survival in Metastatic Melanoma Patients
}

Shang-Jui Wang ${ }^{1}$, Sachin R. Jhawar ${ }^{2}$, Zorimar Rivera-Nunez ${ }^{3}$, Ann W. Silk ${ }^{4}$, John Byun ${ }^{1}$, Eric Miller ${ }^{2}$, Dukagjin Blakaj ${ }^{2}$, Rahul R. Parikh ${ }^{1}$, Joseph Weiner ${ }^{1}$, Sharad Goyal ${ }^{5}$

1. Department of Radiation Oncology, Rutgers Cancer Institute of New Jersey, New Brunswick, USA 2. Department of Radiation Oncology, Ohio State University Comprehensive Cancer Center-James Cancer Hospital and Solove Research Institute, Columbus, USA 3. Department of Biostatistics and Epidemiology, Rutgers School of Public Health, Piscataway, USA 4. Department of Medical Oncology, Dana-Farber Cancer Institute, Boston, USA 5. Department of Radiation Oncology, George Washington University School of Medicine and Health Sciences, Washington, DC, USA

Corresponding author: Sharad Goyal, shgoyal@mfa.gwu.edu

\section{Abstract}

\section{Objective}

Metastatic melanoma patients often receive palliative radiotherapy (RT) and immunotherapy (IT). However, the immunological interplay between RT dose-fractionation and IT is uncertain, and the optimal treatment strategy using RT and IT in metastatic melanoma remains unclear. Our main objective was to examine the effect of RT dose-fractionation on overall survival (OS).

\section{Methods}

Using the National Cancer Database (NCDB), we classified metastatic melanoma patients who received palliative RT into two dose-fractionation groups - conventionally fractionated RT (CFRT; $<5 \mathrm{~Gy} /$ fraction) and hypofractionated RT (HFRT: $\geqslant 5$ Gy/fraction) - with or without IT. Survival analysis was performed using the Cox regression model, Kaplan-Meier method, and propensity-score matching (PSM).

\section{Results}

A total of 5,281 metastatic melanoma patients were included, with a median follow-up of 5.9 months. The three-year OS was highest in patients who received HFRT+IT [37.3\% (95\% CI: 31.1-43.5)] compared to those who received HFRT alone [19.0\% (95\% CI: 16.2-21.9)], CFRT+IT [17.6 (95\%CI: 13.9-21.6)], or CFRT alone [8.6\% (95\%CI: 7.6-9.7); $\mathrm{p}<0.0001]$. The magnitude of OS benefit with the use of IT was greater in those who received HFRT (18.3\%) compared with those who received CFRT $(9.0 \%)(\mathrm{p}<0.0001)$. The addition of IT to HFRT, compared to CFRT, was associated with greater OS benefit in patients treated with RT to the brain and soft tissue/visceral (STV) sites. On PSM analysis, HFRT+IT was associated with improved three-year OS compared to other treatments.

Received 03/12/2020

Review began 03/20/2020 Review ended 06/14/2020 Published 06/22/2020

\section{๑) Copyright 2020}

Wang et al. This is an open access article distributed under the terms of the Creative Commons Attribution License CC-BY 4.0., which permits unrestricted use, distribution, and reproduction in any medium, provided the original author and source are credited.

\section{Conclusion}

Metastatic melanoma patients who received HFRT+IT was associated with the greatest OS benefit. Our findings warrant further prospective evaluation as to whether higher RT dose-per-fraction improves clinical outcomes in metastatic melanoma patients receiving IT.

Categories: Radiation Oncology, Oncology

Keywords: radiotherapy (rt), hypofractionated rt, immunotherapy, metastatic melanoma, dose-fractionation

\section{Introduction}

Melanoma is the first malignancy in which immunotherapy (IT) has gained widespread use in the metastatic setting. Immune checkpoint inhibitors such as anti-cytotoxic T-lymphocyte antigen 4 (CTLA-4) and antiprogrammed cell death-1 (PD-1) antibodies, which are now considered first-line therapies in patients with metastatic melanoma, can reverse the immunosuppressive effects exerted by cancer cells and promote antitumor immunity [1-4]. Despite therapeutic advances with IT, patient outcomes can still be improved as responses tend to be limited to a subset of patients who have preexisting T-cell responses that can be reactivated by immune checkpoint blockade [5].

Nearly half of all patients with metastatic melanoma receive radiotherapy (RT) during their treatment, typically in the setting of oligometastases or for palliation of brain metastases, spinal cord compression, or bleeding tumors. RT has been demonstrated to induce immune-modulation through a variety of mechanisms, including increased presentation of antigens, the release of pro-inflammatory cytokines and molecules, upregulation of death receptors and ligands, and neoantigen formation [6]. These immunogenic 
effects in turn lead to the activation of adaptive antitumor immunity. Thus, the combination of RT with immune checkpoint blockade is a promising therapeutic strategy and has led to the development of clinical trials assessing this combination.

To date, several prospective clinical trials have demonstrated the feasibility, safety, and efficacy of combining IT with RT [7-11]. Recent patterns-of-care studies have also revealed the increasing use of RT and IT in patients with metastatic melanoma $[12,13]$. However, there is no study available to assess the effect of RT dose-fractionation and timing on overall survival (OS) outcomes of combination therapy. To address this gap in the existing literature, we used the National Cancer Database (NCDB) data on metastatic melanoma to further study the interaction between IT and RT. The primary endpoint of this study was to assess whether the use of IT with hypofractionated RT (HFRT) or conventionally fractionated RT (CFRT) in patients with metastatic melanoma was associated with OS. We also examined the effect of RT treatment site and IT timing on OS. Our hypothesis was that the use of HFRT improves OS compared to CFRT in metastatic melanoma patients receiving IT.

\section{Materials And Methods \\ Patient population}

The NCDB is a national, hospital-based registry sponsored by the American College of Surgeons Commission on Cancer $(\mathrm{CoC})$ and the American Cancer Society. It collects information on approximately $70 \%$ of all new invasive cancers diagnosed in the United States annually [14]. Each year, the NCDB receives reports of over one million cancer cases from around 1,500 hospital-based programs accredited by the CoC [14]. The database comprises demographic information, individual diagnosis, and treatment information such as clinical stage, RT dose and volume, and use of IT [15]. The NCDB undergoes extensive internal quality monitoring and validity reviews annually [16]. While the NCDB does not specify the biological agent used for each patient, IT use may include immune checkpoint inhibitors (anti-CTLA-4, anti-PD-1, or anti-PD-L1), interleukins, and oncolytic virus (talimogene laherparepvec), but not BRAF (i.e., dabrafenib or vemurafenib) or MEK (i.e., trametinib) inhibitors.

A total of 19,294 patients diagnosed with stage IV melanoma between 2004-2015 were represented in the database. We excluded patients not receiving RT $(n=12,205)$ or with incomplete or unreliable treatment information (e.g., missing total dose, fraction number, or treatment site) $(n=1,174)$. We classified 5,915 patients who received RT with complete treatment information into CFRT ( $<5$ Gy/fraction) and HFRT ( $\geqslant 5$ Gy/fraction) dose-fractionation groups (Figure 1). For CFRT, we included 3,900 patients who received total doses of RT between 20-70 Gy and $\geqslant 1.5$ Gy/fraction. For HFRT, we included 1,381 patients who received total doses of RT between 15.01-70 Gy and $\leqslant 34$ Gy/fraction, or 10-15 Gy in one fraction. We excluded 634 patients who did not meet the above dose criteria. CFRT and HFRT patients were further divided based on whether they had received IT or not, ultimately resulting in four treatment groups in all (CFRT+IT, CFRT alone, HFRT+IT, and HFRT alone).

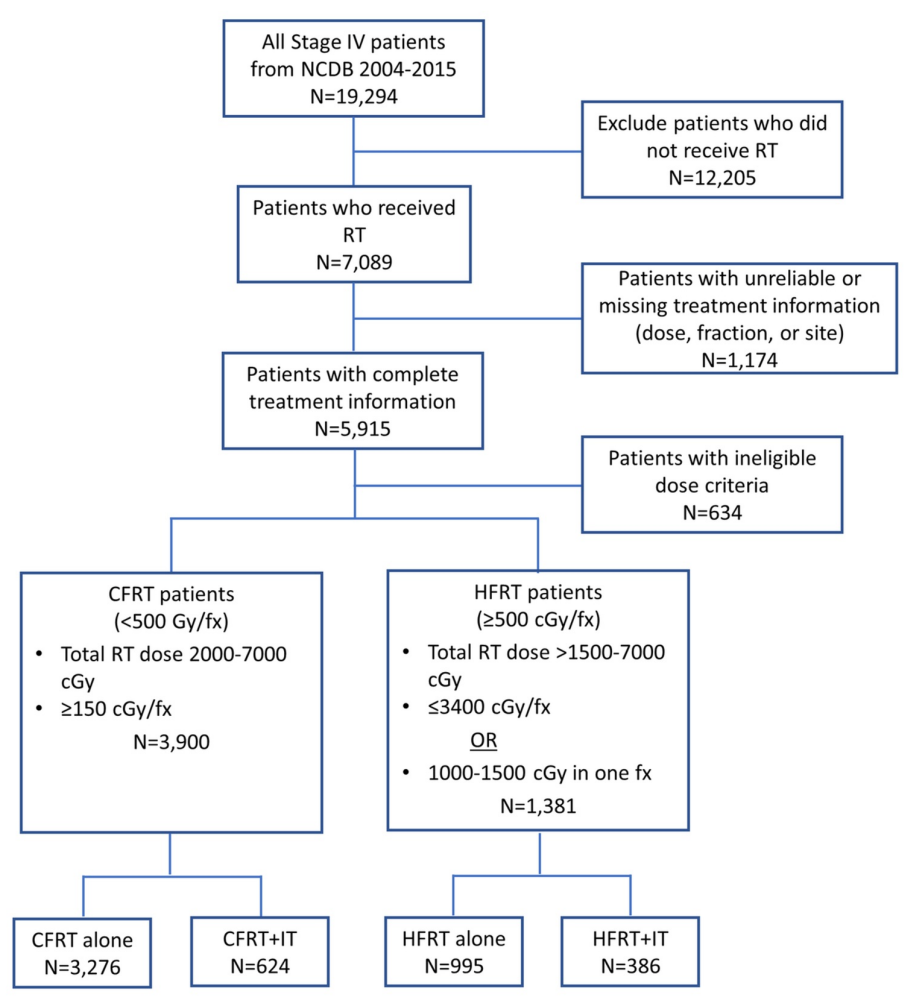


Most patients who received CFRT were treated at 2-3 Gy/fraction, with a median dose of $30 \mathrm{~Gy}$ [interquartile range (IQR): 7.50], and the frequency and total dose distribution at each dose-per-fraction interval are shown in Figure $2 A, 2 B$. For patients who received HFRT, the median dose was 24 Gy (IQR: 10), and the frequency and total dose distribution are shown in Figure 2C, $2 D$.

A

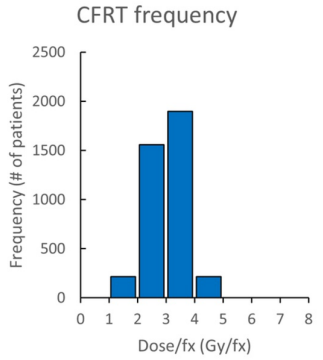

B

CFRT dose distribution

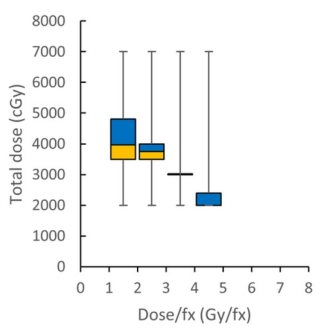

C

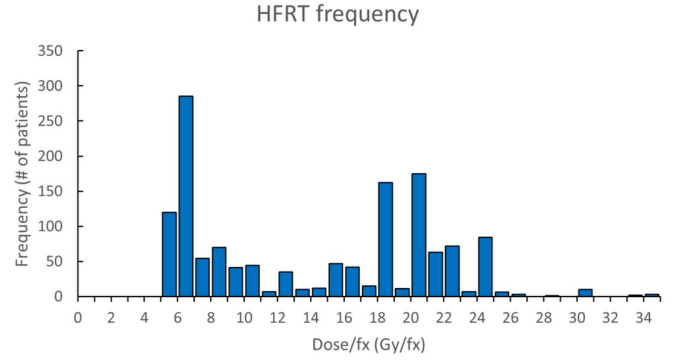

D

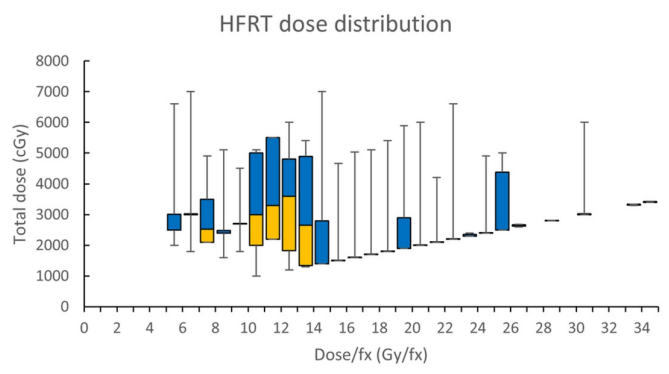

\section{FIGURE 2: Radiotherapy characteristics of the patient cohort}

Histograms depict the frequency of patients who received CFRT (A) and HFRT (C) at various dose-perfraction intervals. Range and distribution of total RT dose are depicted using whisker-box plots for each dose-per-fraction interval in patients who received CFRT (B) and HFRT (D). Dose-per-fraction intervals on the $x$-axis are inclusive of the smaller value, but not the larger value [e.g., in (A), 1,558 CFRT patients received RT between 2.00-2.99 Gy/fraction]. A whisker-box plot consists of a lower box (yellow, 1st quartile - median), an upper box (blue, median - 3rd quartile), and whiskers (minimum and maximum)

CFRT: conventionally fractionated radiotherapy; HFRT: hypofractionated radiotherapy; fx: fraction

\section{Statistical analysis}

Frequencies and proportions were calculated for all demographic and clinical categorical variables. Chisquare and Kruskal-Wallis tests were used to compare proportions and medians. IQRs were calculated to describe median dispersion. Statistical significance was determined at an alpha level of 0.05. Kaplan-Meier survival curves and log-rank statistics were used to examine OS. Survival estimates and 95\% CIs were calculated from survival functions at three years. Survival was calculated from the date of diagnosis to the date of the last contact or confirmed death. Cox proportional hazards regression was used to examine the unadjusted hazard ratio (HR) and adjusted hazard ratios (aHR).

Patient variables included in the multivariable Cox regression model were age, sex, Charlson comorbidity score (CCS), education attainment, median household income, type of health insurance, hospital type, chemotherapy use, any type of surgery, and RT treatment sites [brain, bone, and soft tissue/visceral (STV)].

In a sensitivity analysis, we used one-to-one propensity-score matching (PSM) by modeling individual logistic regressions using treatment groups as dependent variable [HFRT-IT group vs. each of the other three treatment groups (HFRT alone, CFRT-IT, and CFRT alone)] with independent variables including age, sex, race, CCS, type of health insurance, and RT treatment site. Cox proportional hazards regression and KaplanMeier survival curves were used to examine survival between matched groups. All statistical analyses were performed using SAS software version 9.4 (SAS Institute, Cary, NC). 


\section{Cureus}

\section{Results}

\section{Descriptive statistics}

Our analysis examined 5,281 patients with stage IV melanoma who met the inclusion criteria (Figure 1). Of those, $7.3 \%$ of patients received HFRT+IT, $18.8 \%$ received HFRT alone, $11.8 \%$ received CFRT+IT, and $62.0 \%$ received CFRT alone. The mean age was 62.1 years (range: $19-90$ years). Overall, most of the study population was Caucasian (97.0\%), had no comorbidities (CCS: 0) (77.9\%), with a higher proportion of males (69.9\%), and relatively equal distribution of treatments across age groups (Table 1). Patients in the HFRT+IT group had slightly fewer comorbidities (CCS: $>0)(p=0.0014)$ and were more likely to be treated at academic centers $(p<0.0001)$ and to have private insurance $(p<0.0001)$ than those in other treatment groups. Patients who did not receive IT were more likely to receive chemotherapy compared to those who received IT (HFRT/CFRT alone: $25.3 \%$ vs HFRT/CFRT+IT: 10.0\%; p<0.0001).

Patient characteristics

Age (years)

$\leq 50$

$>50-60$

$>60-70$

$>70$

Sex

Female

Male

Race

White

Black

Native American

Asian

Other

Hispanic ethnicity

No

Yes

CCS

0

1

2

Income 2008-12, (\$)

$\geq 62,000$

$\geq 47,999-62,999$

$\geq 38,000-47,999$

$<38,000$

Education attainment ${ }^{\dagger}$

$<7 \%$

138 (36)

7-12.9\%

$13-20.9 \%$

(\%)

85 (22)

267 (69)

377 (98)

$3(0.8)$

$0(0)$

$1(0.3)$

$2(0.5)$

370 (96)

$9(2)$

320 (83)

51 (13)

$15(4)$

167 (43)

119 (31)

73 (19)

25 (6)

145 (38)

74 (19)
HFRT+IT ( $\mathrm{N}=386), \mathrm{n}$

\section{(\%)}

FRT ( $\mathrm{N}=995), \mathrm{n}$

(\%)

(\%)

157 (25)

159 (25)

173 (28)

135 (22)

201 (32)

423 (68)
507 (81)

77 (12)

$40(6)$

608 (98)

7 (1)

2 (0.3)

0 (0)

$5(0.8)$

593 (95)

$15(2)$

931 (94)

$18(2)$

798 (80)

2491 (76)

132 (13)

534 (16)

$65(7)$

251 (8)

230 (37)

195 (31)

119 (19)

75 (12)

$190(30)$

204 (33)

163 (26)
294 (30)

789 (24)

359 (36)

1000 (31)

$261(26)$

923 (28)

219 (22)

818 (25)

140 (14)

465 (14)

318 (32)

1126 (34)

244 (25)

855 (26) 


\section{Cureus}

\begin{tabular}{|c|c|c|c|c|}
\hline$>21 \%$ & $27(7)$ & $62(10)$ & $125(13)$ & $438(13)$ \\
\hline \multicolumn{5}{|l|}{ Insurance } \\
\hline Private insurance & $208(54)$ & $308(49)$ & $427(43)$ & $1263(39)$ \\
\hline Not insured & $13(3)$ & $33(5)$ & $36(4)$ & $188(6)$ \\
\hline Medicaid & $20(5)$ & $47(8)$ & $70(7)$ & $331(10)$ \\
\hline Medicare & $139(36)$ & 209 (33) & $422(42)$ & $1378(42)$ \\
\hline Other government & $3(0.8)$ & $18(3)$ & $21(2)$ & $71(2)$ \\
\hline \multicolumn{5}{|l|}{ Hospital type } \\
\hline Academic/research cancer program & $199(52)$ & 236 (38) & $470(47)$ & $1029(31)$ \\
\hline Community cancer program & $7(2)$ & $46(7)$ & $28(3)$ & $302(9)$ \\
\hline $\begin{array}{l}\text { Comprehensive community cancer } \\
\text { program }\end{array}$ & $95(25)$ & $232(37)$ & $283(28)$ & $1405(43)$ \\
\hline Integrated network cancer program & $49(13)$ & $55(9)$ & $152(15)$ & $325(10)$ \\
\hline \multicolumn{5}{|l|}{ Chemotherapy } \\
\hline No & $351(91)$ & $556(89)$ & $722(72)$ & $2448(75)$ \\
\hline Yes & $35(9)$ & $66(11)$ & $268(27)$ & $814(25)$ \\
\hline \multicolumn{5}{|l|}{ Any type of surgery } \\
\hline No & $303(78)$ & $508(81)$ & $778(78)$ & $2678(82)$ \\
\hline Yes & $83(22)$ & $116(19)$ & $216(22)$ & $592(18)$ \\
\hline \multicolumn{5}{|l|}{ RT treatment site } \\
\hline Brain & $273(71)$ & $323(52)$ & $689(69)$ & $2102(64)$ \\
\hline Bone & $33(9)$ & 119 (19) & $62(6)$ & 467 (14) \\
\hline STV sites & $80(21)$ & $182(29)$ & 244 (25) & $707(22)$ \\
\hline
\end{tabular}

TABLE 1: Demographic and clinical characteristics of stage IV melanoma patients $(n=5,281)$ in the NCDB 2004-2015

†Proportion of the number of adults in the patient's zip code who did not graduate from high school

RT: radiotherapy; IT: immunotherapy; HFRT: hypofractionated radiotherapy; CFRT: conventionally fractionated radiotherapy; CCS: Charlson/Deyo comorbidity score; STV: soft tissue/visceral; NCDB: National Cancer Database

\section{Survival analysis}

In univariate models, most patient characteristics were significantly associated with the risk of dying, except for race, income, and chemotherapy use (Table 2). On multivariate analysis, age of $>60$ years (aHR range: $1.20-1.60$ ), male sex (aHR: 1.11, 95\% CI: 1.03-1.20; $\mathrm{p}=0.0061$ ), and CCS of $\geqslant 1$ (aHR range: $1.19-1.22$ ) were associated with increased risk of death. Patients who were not insured (aHR: 1.28, 95\% CI: 1.09-1.51; $\mathrm{p}=0.0029$ ) or on Medicaid (aHR: 1.32, 95\% CI: $1.16-1.51$; $\mathrm{p}<0.0001$ ) had higher risk of dying compared to patients on private insurance. However, the most significant factor associated with the risk of dying on multivariate analysis was the type of treatment received. Patients who received CFRT alone (aHR: 2.81, 95\% CI: 2.36-3.34; p<0.0001), CFRT+IT (aHR: 1.95, 95\% CI: 1.59-2.39; p<0.0001), and HFRT alone (HR: $1.57,95 \%$ CI: $1.31-1.89 ; \mathrm{p}<0.0001$ ) were all at higher risk of dying compared to patients treated with HFRT+IT.

\begin{tabular}{|l|l|l|l|}
\hline Patient characteristics & Univariate HR $(95 \% \mathrm{Cl})$ & P-value & Multivariate HR (95\% Cl) \\
\hline $\begin{array}{l}\text { Type of treatment } \\
\text { HFRT+IT }\end{array}$ & REF & REF \\
\hline
\end{tabular}




\section{Cureus}

\begin{tabular}{|c|c|c|c|c|}
\hline CFRT+IT & $1.73(1.44-2.09)$ & $<0.0001$ & $1.95(1.59-2.39)$ & $<0.0001$ \\
\hline HFRT alone & $1.68(1.42-2.00)$ & $<0.0001$ & $1.57(1.31-1.89)$ & $<0.0001$ \\
\hline CFRT alone & $2.87(2.45-3.36)$ & $<0.0001$ & $2.81(2.36-3.34)$ & $<0.0001$ \\
\hline \multicolumn{5}{|l|}{ Age (years) } \\
\hline$\leq 50$ & REF & & REF & \\
\hline$>50-60$ & $1.09(0.99-1.20)$ & 0.0824 & $1.08(0.96-1.20)$ & 0.2000 \\
\hline$>60-70$ & 1.19 (1.09-1.31) & 0.0002 & $1.20(1.06-1.35)$ & 0.0035 \\
\hline$>70$ & $1.53(1.40-1.67)$ & $<0.0001$ & $1.60(1.40-1.82)$ & $<0.0001$ \\
\hline \multicolumn{5}{|l|}{ Sex } \\
\hline Female & REF & & & \\
\hline Male & $1.15(1.08-1.24)$ & $<0.0001$ & $1.11(1.03-1.20)$ & 0.0061 \\
\hline \multicolumn{5}{|l|}{ Race } \\
\hline White & REF & & REF & \\
\hline Black & $1.02(0.78-1.34)$ & 0.8761 & - & - \\
\hline Native American & $0.96(0.40-2.30)$ & 0.9238 & - & - \\
\hline Asian & $0.69(0.40-1.19)$ & 0.1792 & - & - \\
\hline Other & $0.93(0.58-1.50)$ & 0.7635 & - & - \\
\hline \multicolumn{5}{|l|}{ Hispanic ethnicity } \\
\hline No & REF & & REF & \\
\hline Yes & $0.76(0.61-0.95)$ & 0.0168 & $0.84(0.65-1.08)$ & 0.1737 \\
\hline \multicolumn{5}{|l|}{$\operatorname{ccs}$} \\
\hline 0 & REF & & REF & \\
\hline 1 & 1.24 (1.13-1.35) & $<0.0001$ & $1.19(1.08-1.30)$ & 0.0003 \\
\hline 2 & $1.43(1.27-1.62)$ & $<0.0001$ & $1.22(1.07-1.39)$ & 0.0036 \\
\hline \multicolumn{5}{|l|}{ Income 2008-12, (\$) } \\
\hline$\geq 62,000$ & REF & & REF & \\
\hline$\geq 47,999-62,999$ & $1.05(0.97-1.14)$ & 0.2498 & - & - \\
\hline$\geq 38,000-47,999$ & $1.05(0.96-1.14)$ & 0.2775 & - & - \\
\hline$<38,000$ & $1.07(0.96-1.18)$ & 0.2223 & - & - \\
\hline \multicolumn{5}{|c|}{ Education attainment ${ }^{\dagger}$} \\
\hline$<7 \%$ & REF & & REF & \\
\hline $7-12.9 \%$ & $1.10(1.01-1.19)$ & 0.0244 & $1.03(0.92-1.16)$ & 0.6182 \\
\hline $13-20.9 \%$ & $1.01(0.93-1.11)$ & 0.7887 & 0.94 (0.86-1.04) & 0.2104 \\
\hline$>21 \%$ & $1.07(0.96-1.19)$ & 0.2008 & $1.07(0.98-1.17)$ & 0.1347 \\
\hline \multicolumn{5}{|l|}{ Insurance } \\
\hline Private insurance & REF & & REF & \\
\hline Not insured & $1.25(1.09-1.45)$ & 0.0021 & $1.28(1.09-1.51)$ & 0.0029 \\
\hline Medicaid & $1.27(1.13-1.42)$ & $<0.0001$ & $1.32(1.16-1.51)$ & $<0.0001$ \\
\hline Medicare & $1.38(1.29-1.48)$ & $<0.0001$ & $1.07(0.97-1.18)$ & 0.1950 \\
\hline
\end{tabular}




\section{Cureus}

\begin{tabular}{|c|c|c|c|c|}
\hline Other government & $1.04(0.83-1.30)$ & 0.7265 & $0.88(0.68-1.12)$ & 0.2935 \\
\hline \multicolumn{5}{|l|}{ Hospital type } \\
\hline Academic/research cancer program & REF & & REF & \\
\hline Community cancer program & $1.50(1.33-1.70)$ & $<0.0001$ & $1.24(1.08-1.42)$ & 0.0018 \\
\hline Comprehensive community cancer program & $1.33(1.23-1.43)$ & $<0.0001$ & $1.16(1.07-1.25)$ & 0.0003 \\
\hline Integrated network cancer program & $1.21(1.09-1.35)$ & 0.0004 & $1.14(1.02-1.28)$ & 0.0254 \\
\hline \multicolumn{5}{|l|}{ Chemotherapy } \\
\hline No & REF & & REF & \\
\hline Yes & $1.02(0.95-1.10)$ & 0.5776 & - & - \\
\hline \multicolumn{5}{|l|}{ Any type of surgery } \\
\hline No & REF & & REF & \\
\hline Yes & $0.72(0.66-0.78)$ & $<0.0001$ & $0.82(0.75-0.89)$ & $<0.0001$ \\
\hline \multicolumn{5}{|l|}{ RT treatment site } \\
\hline STV sites & REF & & REF & \\
\hline Brain & $1.58(1.46-1.71)$ & $<0.0001$ & $1.75(1.60-1.91)$ & $<0.0001$ \\
\hline Bone & $1.57(1.41-1.76)$ & $<0.0001$ & $1.52(1.35-1.72)$ & $<0.0001$ \\
\hline
\end{tabular}

TABLE 2: Univariate and multivariate Cox regression analysis of individual demographic and clinical characteristics with overall survival in the population receiving $R T(n=5,281)$

†Proportion of the number of adults in the patient's zip code who did not graduate from high school

RT: radiotherapy; IT: immunotherapy; HFRT: hypofractionated radiotherapy; CFRT: conventionally fractionated radiotherapy; CCS: Charlson/Deyo comorbidity score; STV: soft tissue/visceral; REF: reference; HR: hazard ratio; Cl: confidence interval

The median follow-up time for the cohort was 5.9 (IQR: 11.4) months. Overall, HFRT+IT patients had the highest three-year OS [37.3\% (95\% CI: 31.1-43.5)] compared to HFRT alone [19.0\% (95\% CI: 16.2-21.9)], CFRT+IT [17.6\% (95\% CI: 13.9-21.6)], and CFRT alone [8.6\% (95\% CI: 7.6-9.7), overall log rank: <0.0001] (Figure 3). HFRT+IT group had a significantly higher three-year OS compared to the CFRT+IT group $(\mathrm{p}<0.0001)$. Compared to RT-alone patients, the addition of IT was associated with improved three-year OS in both HFRT (HFRT alone vs. HFRT+IT, $\mathrm{p}<0.0001$ ) and CFRT (CFRT alone vs. CFRT+IT, $\mathrm{p}<0.0001$ ) treatment groups. However, the magnitude of OS benefit associated with IT was significantly larger in the HFRT group (three-year $\triangle$ OSIT $=18.3 \%$ ) compared to the CFRT group (three-year $\triangle \mathrm{OSIT}=9.0 \%, \mathrm{p}<0.0001$ ) (Figure 3). 


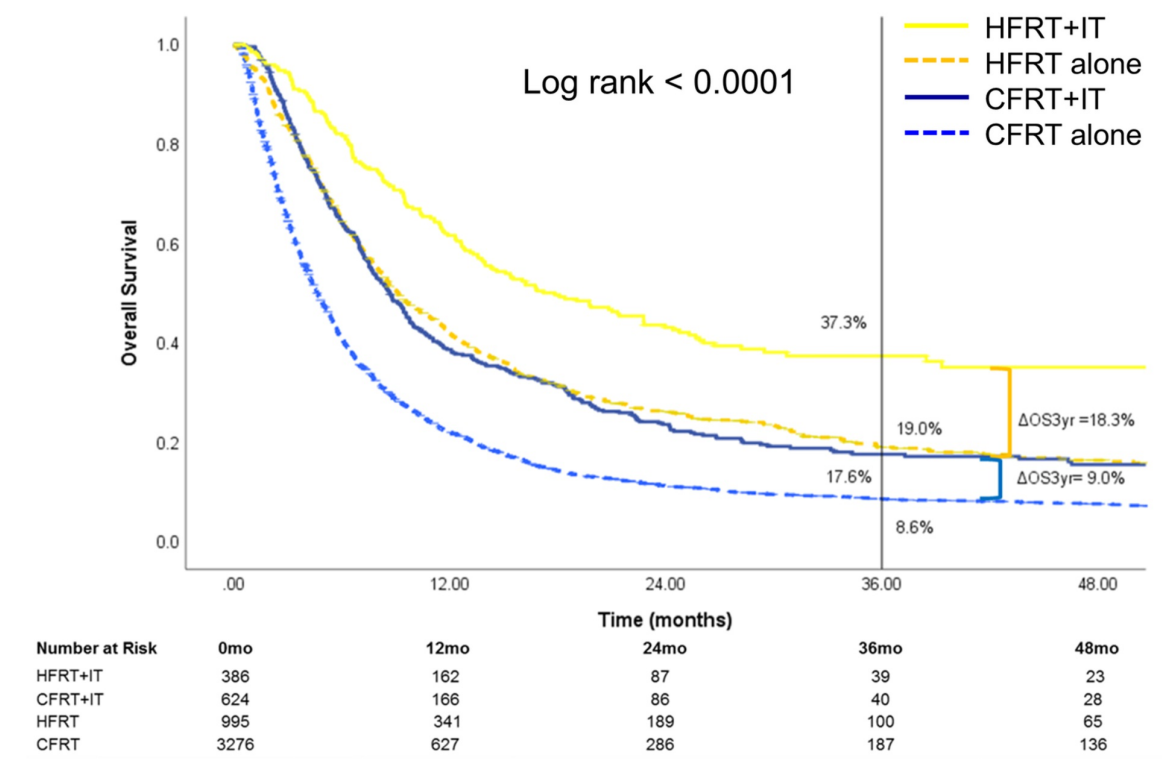

FIGURE 3: Kaplan-Meier curves for overall survival for entire cohort

The entire cohort was stratified by treatment received

HFRT: hypofractionated radiotherapy; CFRT: conventionally fractionated radiotherapy; IT: immunotherapy; OS: overall survival

Stratification by RT treatment site (Figures 4-6) showed that the survival benefit of HFRT+IT was evident for brain and STV metastatic sites. For patients treated for metastatic brain sites, three-year OS was highest for HFRT+IT [34.6\% (95\% CI: 27.1-42.3)] compared to HFRT alone [18.4\% (95\% CI: 15.2-21.9)], CFRT+IT [12.5\% (95\% CI: 8.0-18.0)], or CFRT alone [5.6\% (95\% CI: 4.6-6.8)] (Figure 4). For those treated for metastatic STV sites, three-year OS was also highest for HFRT+IT [45.9\% (95\% CI: 33.1-57.8)] compared to HFRT alone [19.0\% (95\% CI: 13.7-25.1)], CFRT+IT [24.8\% (95\% CI: 17.8-32.4)], or CFRT alone [18.8\% (95\% CI: 15.7-22.1)] (Figure 5). The OS benefit with the addition of IT was significantly greater in the HFRT group compared to the CFRT group for patients who received RT to the brain (three-year $\triangle$ OSIT: HFRT vs CFRT, $16.2 \%$ vs $6.9 \%$, $\mathrm{p}<0.0001$ ) or STV sites (three-year $\triangle$ OSIT: HFRT vs CFRT, $26.9 \%$ vs $6.0 \%$, $\mathrm{p}<0.0001$ ) sites. On the other hand, while treatment with HFRT+IT in patients who received RT to bone sites was associated with the highest OS, the addition of IT to HFRT did not significantly improve survival (Figure 6).

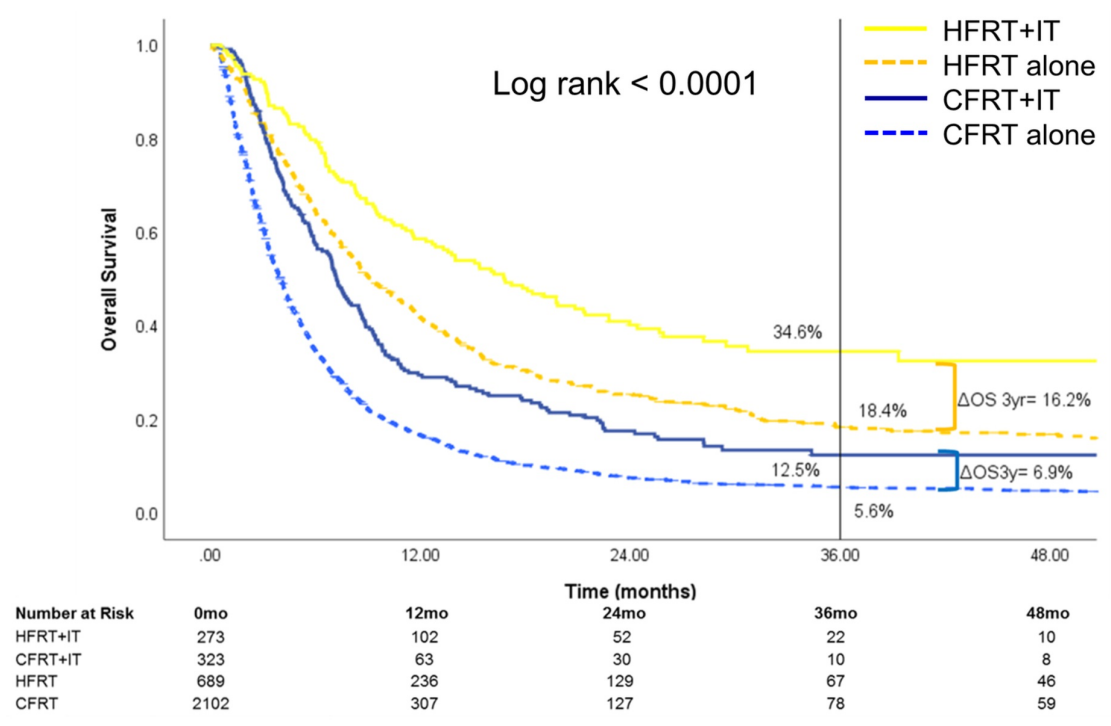

FIGURE 4: Overall survival of patients who received RT to the brain 


\section{Cureus}

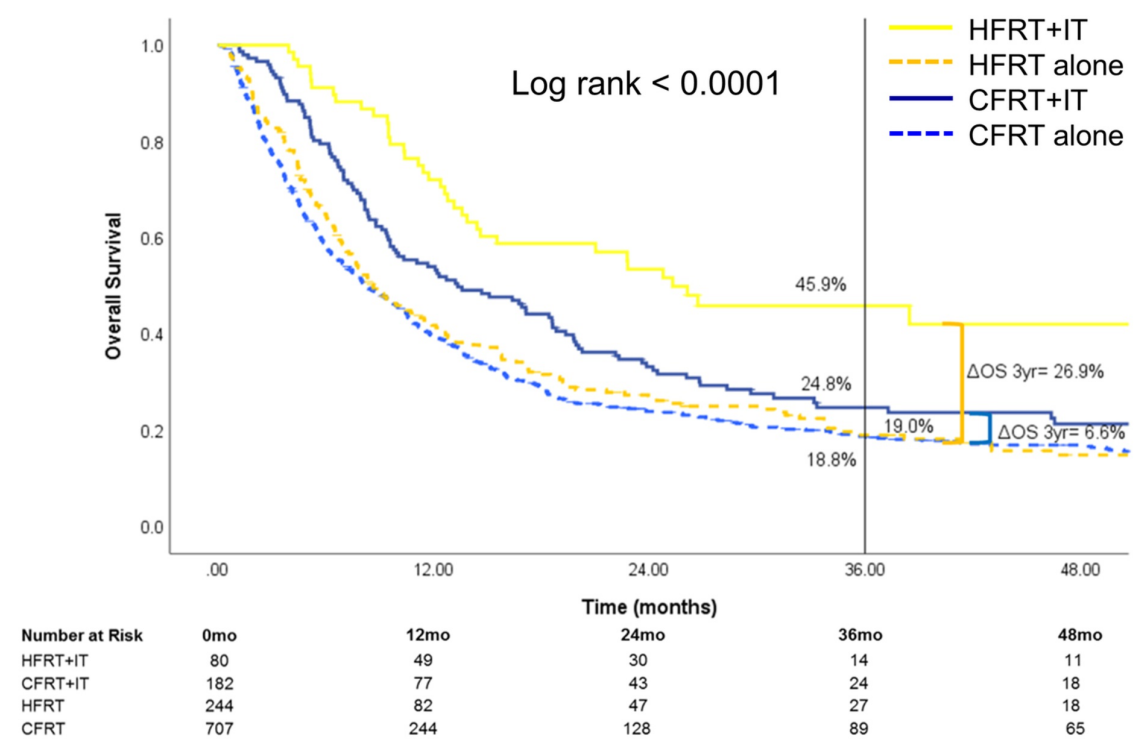

FIGURE 5: Overall survival of patients who received RT to STV sites

The subset of patients who received radiotherapy to STV sites was stratified by treatment received

HFRT: hypofractionated radiotherapy; CFRT: conventionally fractionated radiotherapy; IT: immunotherapy; STV: soft tissue/visceral; OS: overall survival

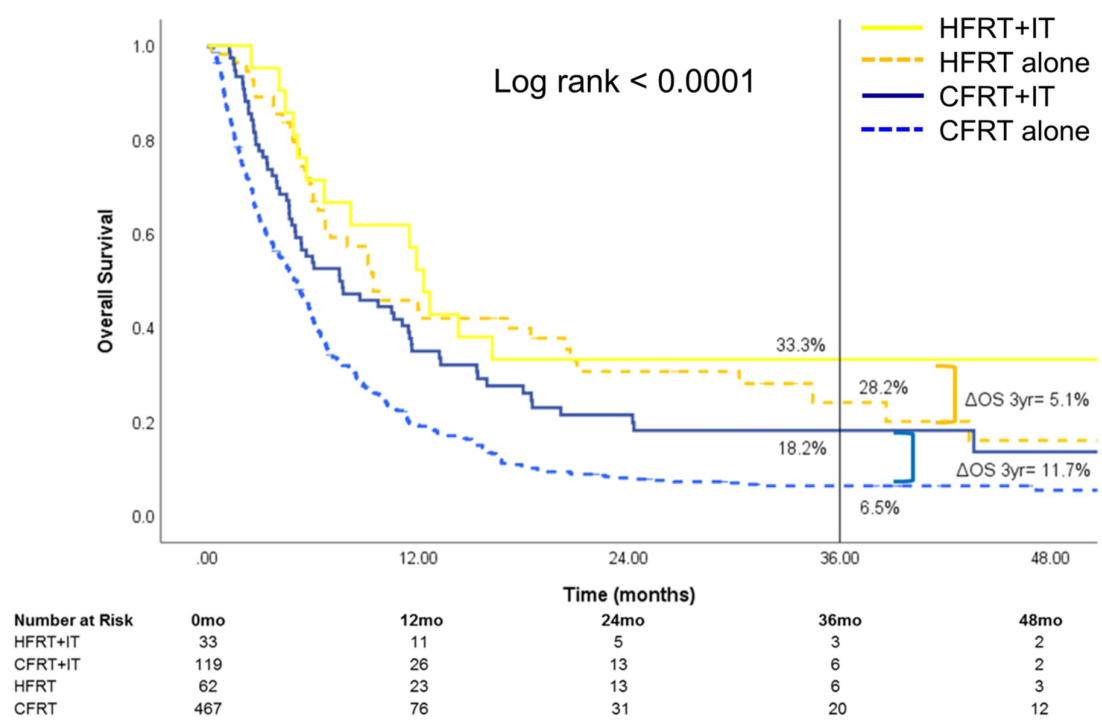

FIGURE 6: Overall survival of patients who received RT to the bone

The subset of patients who received radiotherapy to the bone was stratified by treatment received

HFRT: hypofractionated radiotherapy; CFRT: conventionally fractionated radiotherapy; IT: immunotherapy; OS: overall survival 


\section{Cureus}

After PSM, 1,200 patients ( $\mathrm{n}=300$ per treatment group) were available for analysis (Table 3). Treatment with HFRT+IT continued to be associated with superior three-year OS [36.6\% (95\% CI: 30.0-43.5)] compared to HFRT alone [20.4\% (95\% CI: 15.4-25.9)], CFRT+IT [16.0\% (95\% CI: 10.9-21.9)], and CFRT alone [6.5\% (95\% CI: 3.9-10.0)]. OS benefit associated with the utility of IT remained significantly greater in the HFRT group (three-year $\Delta$ OSIT $=16.2 \%$ ) compared with the CFRT group (three-year $\triangle \mathrm{OSIT}=9.5 \%, \mathrm{p}<0.0001$ ) (Figure 7 and Table 4).

\begin{tabular}{|c|c|c|c|c|}
\hline Patient characteristics & HFRT+IT, n (\%) & CFRT+IT, n (\%) & HFRT alone, $n$ (\%) & CFRT alone, $n$ (\%) \\
\hline \multicolumn{5}{|l|}{ Age (years) } \\
\hline$\leq 50$ & $70(23)$ & $70(23)$ & $70(23)$ & $68(23)$ \\
\hline$>50-60$ & $82(27)$ & $82(27)$ & $82(27)$ & $84(27)$ \\
\hline$>60-70$ & $76(25)$ & $76(25)$ & $76(25)$ & 76 (25) \\
\hline$>70$ & $72(24)$ & $72(24)$ & $72(24)$ & $72(24)$ \\
\hline \multicolumn{5}{|l|}{ Sex } \\
\hline Female & $84(28)$ & $84(28)$ & $84(28)$ & $81(27)$ \\
\hline Male & 216 (72) & $216(72)$ & 216 (72) & $219(73)$ \\
\hline \multicolumn{5}{|l|}{ Race } \\
\hline White & $299(100)$ & $299(100)$ & $299(100)$ & $299(100)$ \\
\hline Others & $1(0)$ & $1(0)$ & $1(0)$ & $1(0)$ \\
\hline \multicolumn{5}{|l|}{$\operatorname{ccs}$} \\
\hline 0 & $10(3)$ & $10(3)$ & $10(3)$ & $10(3)$ \\
\hline 1 & $29(10)$ & $29(10)$ & $29(10)$ & $29(10)$ \\
\hline 2 & $261(87)$ & $261(87)$ & $261(87)$ & $261(87)$ \\
\hline \multicolumn{5}{|l|}{ Insurance } \\
\hline Private insurance & $176(59)$ & $176(59)$ & $176(59)$ & $176(59)$ \\
\hline Not insured & $5(2)$ & $5(2)$ & $5(2)$ & $5(2)$ \\
\hline Medicaid & $10(3)$ & $10(3)$ & $10(3)$ & $10(3)$ \\
\hline Medicare & $108(36)$ & $108(36)$ & $108(36)$ & 108 (36) \\
\hline Other government & $1(0.3)$ & $1(0.3)$ & $1(0.3)$ & $1(0.3)$ \\
\hline \multicolumn{5}{|l|}{ Site treated } \\
\hline Brain & $218(72)$ & $218(72)$ & $218(72)$ & $218(72)$ \\
\hline Bone & $24(8)$ & $24(8)$ & $24(8)$ & $24(8)$ \\
\hline STV sites & 58 (19) & 58 (19) & 58 (19) & 58 (19) \\
\hline
\end{tabular}

TABLE 3: Propensity-score-matched population for stage IV melanoma patients $(n=1,200)$ in the NCDB 2004-2015

RT: radiotherapy; IT: immunotherapy; HFRT: hypofractionated radiotherapy; CFRT: conventionally fractionated radiotherapy; CCS: Charlson/Deyo comorbidity score; STV: soft tissue/visceral; NCDB: National Cancer Database 


\section{Cureus}

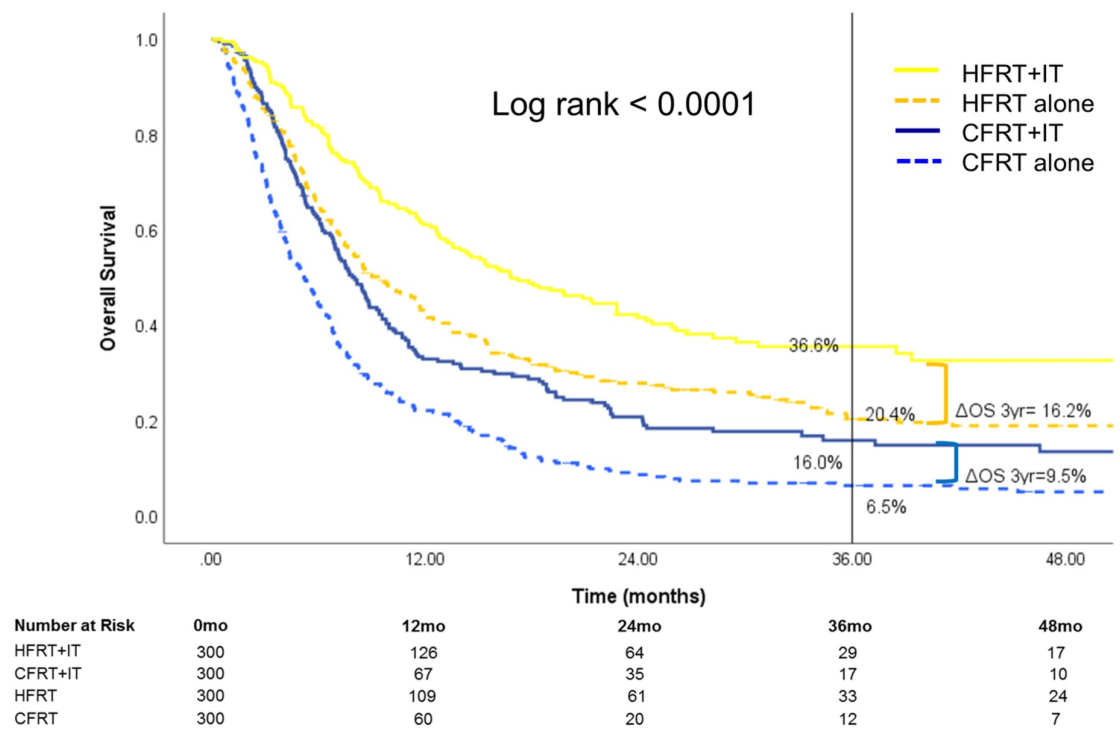

FIGURE 7: Overall survival after propensity-score matching of 1,200 patients ( $n=300$ for each treatment group)

HFRT: hypofractionated radiotherapy; CFRT: conventionally fractionated radiotherapy; IT: immunotherapy; OS: overall survival

\begin{tabular}{|l|l|l|}
\hline Patient characteristics & Univariate HR $(95 \% \mathrm{Cl})$ & P-value \\
\hline Type of treatment & REF & $<0.01$ \\
\hline HFRT+IT & $1.86(1.48-2.33)$ & $<0.01$ \\
CFRT+IT & $1.57(1.26-1.96)$ & $<0.01$
\end{tabular}

\section{TABLE 4: Univariate Cox regression analysis for propensity-score-matched population}

RT: radiotherapy; IT: immunotherapy; HFRT: hypofractionated radiotherapy; CFRT: conventionally fractionated radiotherapy; REF: reference; HR: hazard ratio; $\mathrm{Cl}$ : confidence interval

Of patients treated with both RT and IT, survival was similar regardless of IT timing [neoadjuvant IT (IT > 30 days prior to RT), concurrent IT (IT within 30 days of RT) or adjuvant IT (IT >30 days after RT)] (Figure 8). After stratification by site treated, patients who received treatment to the brain showed a difference in survival that was marginally significant in favor of concurrent/adjuvant IT (log rank: 0.0749) (Figure 9). Conversely, survival was similar in patients who received treatment to STV sites or bone when stratified by IT timing (Figures 10, 11). 


\section{Cureus}

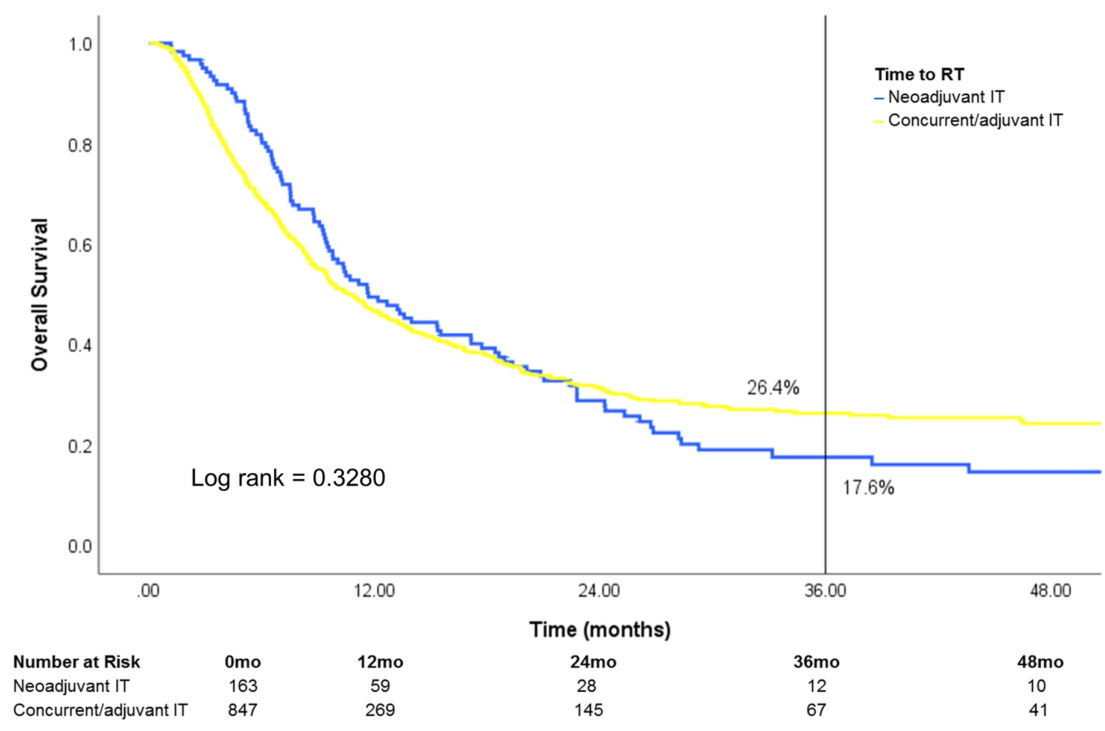

FIGURE 8: Subset analysis of IT timing on overall survival

Patients who received both RT and IT $(n=1,010)$ were stratified by the timing of initiating IT relative to RT RT: radiotherapy; IT: immunotherapy

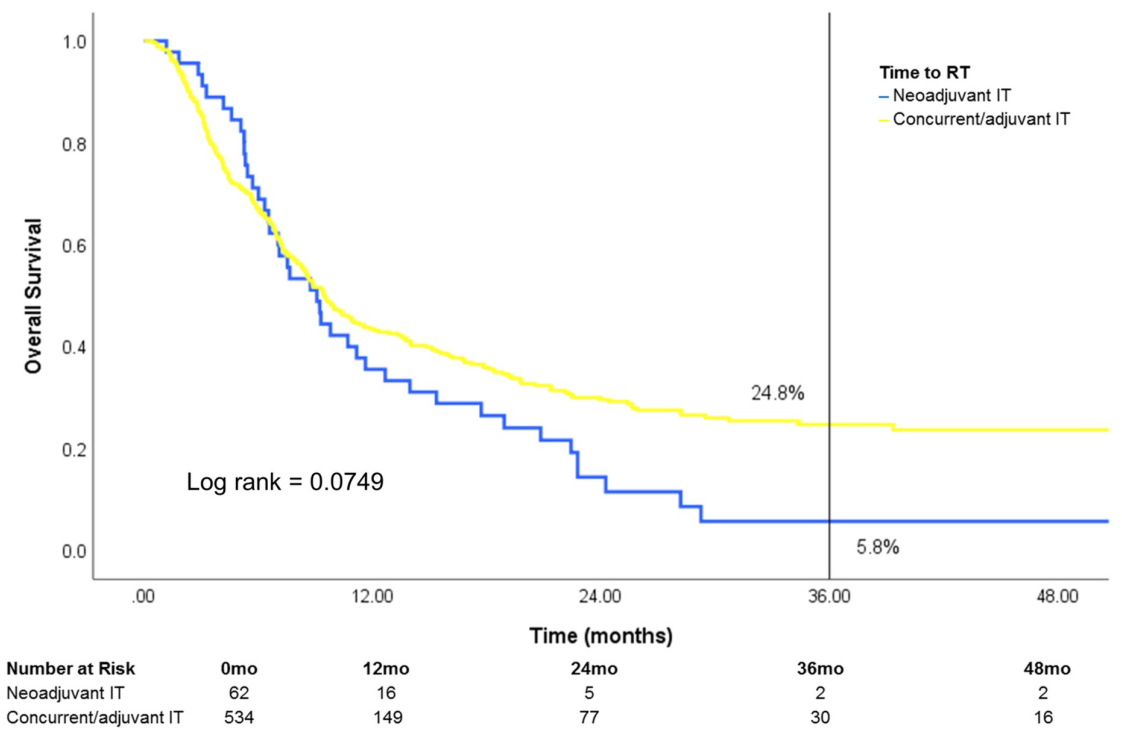

FIGURE 9: Effect of IT timing on overall survival in patients who received $R T$ to the brain

Patients who received RT to the brain and IT ( $n=596)$ were stratified by IT timing

RT: radiotherapy; IT: immunotherapy 


\section{Cureus}

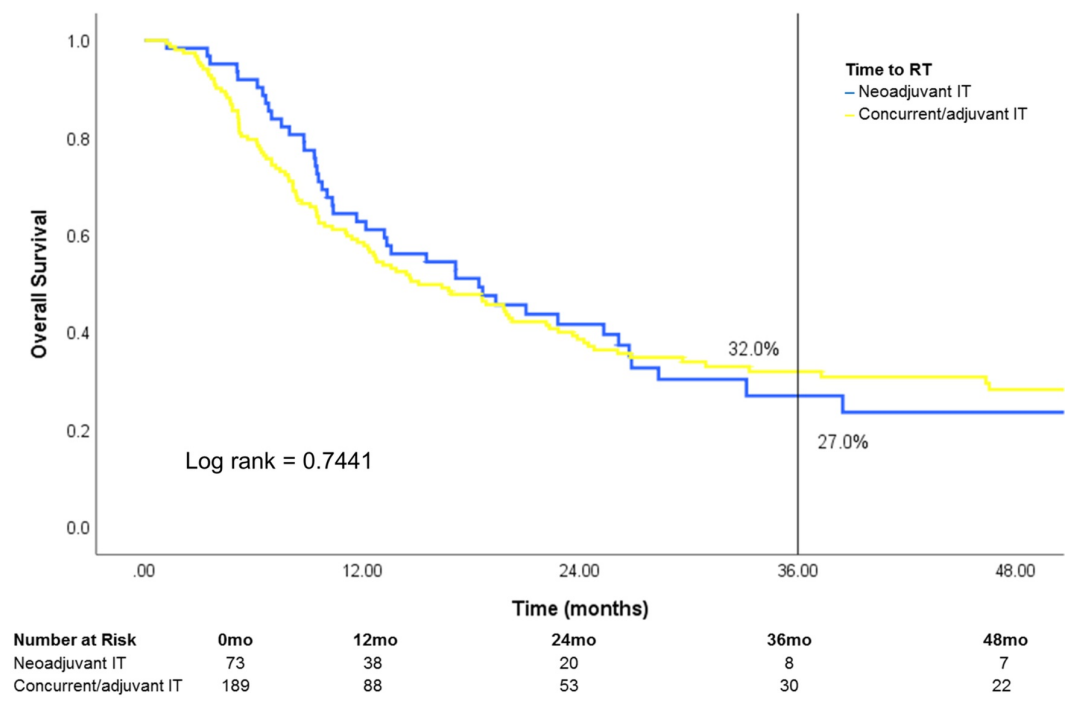

FIGURE 10: Effect of IT timing on overall survival in patients who received RT to STV sites

Patients who received RT to STV sites and IT $(n=262)$ were stratified by IT timing

RT: radiotherapy; IT: immunotherapy; STV: soft tissue/visceral

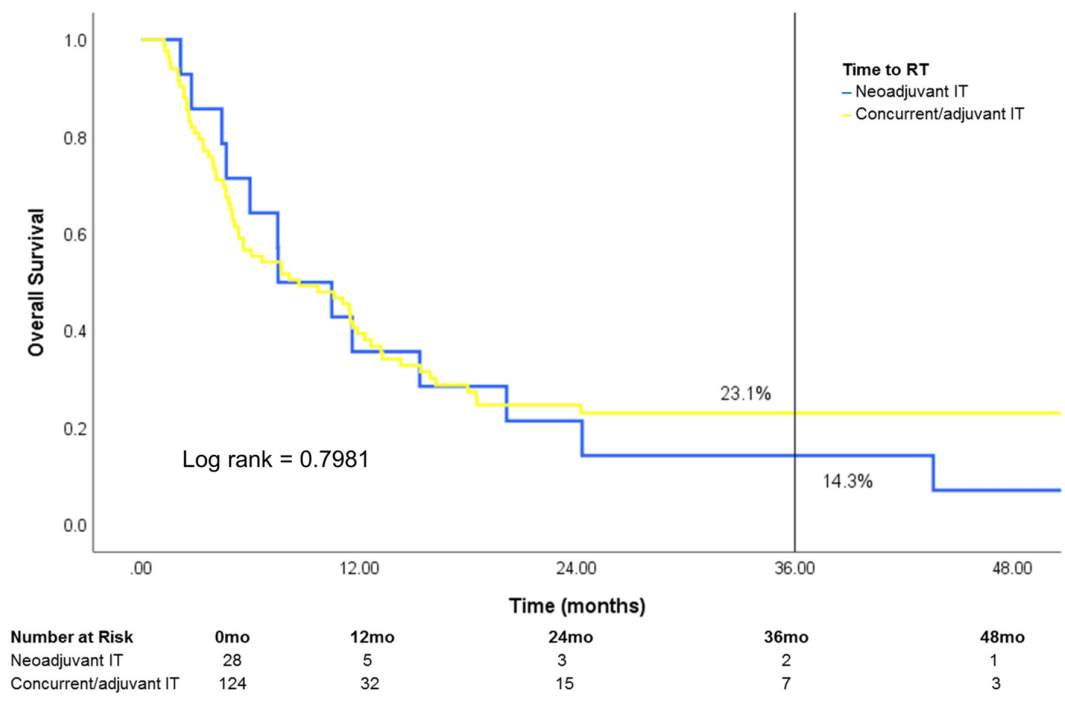

FIGURE 11: Effect of IT timing on overall survival in patients who received $R T$ to the bone

Patients who received RT to the bone and IT ( $n=152)$ were stratified by IT timing

RT: radiotherapy; IT: immunotherapy

\section{Discussion}

For patients receiving IT for metastatic melanoma, RT treatment with HFRT was associated with superior survival compared to CFRT. Moreover, patients treated with HFRT was associated with significantly greater survival benefit from the use of IT compared to those treated with CFRT, especially patients treated with RT to the brain and STV sites. We obtained similar findings after PSM analysis, including matching by irradiated sites. 
Currently, there is no evidence to guide the optimal dose-fractionation of RT in combination with IT for the treatment of any malignancy. There are only a handful of ongoing clinical trials evaluating RT fraction size in combination with IT [17]. However, available clinical evidence has shown that RT with higher dose-perfraction, when combined with IT, results in favorable outcomes in metastatic melanoma. Several studies have reported evidence of the abscopal effect with stereotactic body RT (SBRT) in metastatic melanoma patients receiving IT [18-20]. Moreover, in a recent systemic review of studies reporting the therapeutic efficacy of combined RT and ipilimumab in metastatic melanoma, patients who received higher doses-perfraction (>3 Gy) showed better clinical outcomes [21]. Similarly, preclinical evidence skews toward HFRT/SBRT fraction sizes to be more effective in providing therapeutic synergy with IT [17].

In this study, we showed that metastatic melanoma patients who received IT had significantly higher OS if they received HFRT ( $>5 \mathrm{~Gy} /$ fraction) rather than CFRT ( $\leqslant 5 \mathrm{~Gy} /$ fraction). Moreover, we further ascertained the survival contribution of IT in patients receiving palliative RT by comparing patients treated with HFRT or CFRT, with or without IT. Although patients receiving either CFRT or HFRT were associated with significant improvement in three-year OS when IT was also given compared to their counterparts who did not receive IT, the absolute survival benefit from IT in patients receiving HFRT was greater than those receiving CFRT. As such, our analyses suggest that there is a differential effect with different RT dose-fractionations, with the combination of HFRT and IT potentially exhibiting greater therapeutic effect that translates into improved survival. One possible reason for this observation of ours is that patients who were treated with CFRT for palliation may potentially benefit less from IT. Greater number of RT fractions and a generally larger area of treatment with CFRT may lead to more substantial lymphopenia, which can hinder tumor cell eradication by cytotoxic T lymphocytes [22-24]. Another possibility is that patients receiving HFRT may benefit more from IT, perhaps due to the synergistic effects of higher dose-per-fraction with checkpoint inhibition, as previously discussed [17-20].

The treatment site is likely an important determinant of the response to therapy. It has been suggested that RT to lungs and liver is more immunogenic than to other organs [25,26]. Furthermore, although brain parenchyma was previously considered to be immune-privileged, recent evidence has shown that immune cells can cross the blood-brain barrier, and brain metastases from melanoma respond to immune checkpoint inhibitors [27-29]. Consistent with this, our analysis demonstrated that patients with metastatic brain or STV lesions demonstrated a greater survival benefit with the addition of IT to HFRT compared to those with osseous metastatic lesions.

There are several limitations to this study. First, differential misclassification may still exist toward HFRT being a more effective treatment than CFRT against metastatic melanoma using the NCDB database. For example, patients treated with radiosurgery may have had an intracranial disease of lower volume than those who received whole-brain RT; similarly, patients treated with CFRT to the bones were more likely to harbor diffuse osseous metastases than those who underwent SBRT. Additionally, CFRT may be less effective in eradicating melanoma cells, which are less radiosensitive with a lower $\alpha / \beta$ ratio [30]. Finally, HFRT dosing can often achieve a higher biologically effective dose to the tumor lesions and result in greater tumoricidal effect. To circumvent these biases, we minimized the potential for misclassification by comparing HFRT or CFRT patients who received IT with their respective counterparts who did not. As such, we were able to determine the benefit derived from IT in HFRT or CFRT patients separately, rather than directly comparing HFRT patients with CFRT patients. Second, this is a retrospective study that utilized data collected only from facilities accredited by the $\mathrm{CoC}$, which may generally include patients who received higher quality care and had better outcomes. Third, while we adjusted our models for important covariates, we were unable to account for factors that were not available in the database, including total disease burden, size and number of lesions treated by RT, or the type of immunotherapeutic agents utilized. Finally, we were unable to exclude the possibility that patients may have received multiple courses of RT to the same or different disease sites.

Despite these limitations, our study has several strengths. In addition to defining the two dose-fractionation criteria by a commonly-used threshold of $5 \mathrm{~Gy}$ /fraction, we only included patients who received clinically meaningful doses of RT at any given dose-per-fraction to ensure that we captured the appropriate cohort for our analysis. Furthermore, we examined the survival differences imparted by IT by including HFRT-/CFRTalone groups in our four-arm analysis. Additionally, our results after PSM were very similar to that of the original analysis, indicating that selection bias had a minimal effect on our analysis.

\section{Conclusions}

Our analysis demonstrated that patients treated with IT in combination with higher dose-per-fraction using HFRT had superior OS compared to those treated with IT and CFRT. Furthermore, we observed a differential effect of IT on RT fractionation, with significantly greater improvement in OS when IT was utilized in patients treated with HFRT compared to CFRT, especially in patients who received RT to the brain and STV sites. Altogether, we believe our study contributes to the literature by addressing a critical gap of knowledge regarding the optimal dose-fractionation of palliative RT for metastatic melanoma in conjunction with IT, thereby underscoring the significance of and providing potential guidance for future prospective studies addressing this issue. Furthermore, designing trials by utilizing specific immunotherapeutic agents is essential to better evaluate the interactive effects with RT. 


\section{Additional Information \\ Disclosures}

Human subjects: Consent was obtained by all participants in this study. Animal subjects: All authors have confirmed that this study did not involve animal subjects or tissue. Conflicts of interest: In compliance with the ICMJE uniform disclosure form, all authors declare the following: Payment/services info: All authors have declared that no financial support was received from any organization for the submitted work. Financial relationships: All authors have declared that they have no financial relationships at present or within the previous three years with any organizations that might have an interest in the submitted work. Other relationships: All authors have declared that there are no other relationships or activities that could appear to have influenced the submitted work.

\section{References}

1. Hodi FS, O'Day SJ, McDermott DF, et al.: Improved survival with ipilimumab in patients with metastatic melanoma. N Engl J Med. 2010, 363:711-723. 10.1056/NEJMoa1003466

2. Topalian SL, Sznol M, McDermott DF, et al.: Survival, durable tumor remission, and long-term safety in patients with advanced melanoma receiving nivolumab. J Clin Oncol. 2014, 32:1020-1030. 10.1200/JCO.2013.53.0105

3. Robert C, Long GV, Brady B, et al.: Nivolumab in previously untreated melanoma without BRAF mutation . N Engl J Med. 2015, 372:320-330. 10.1056/NEJMoa1412082

4. Larkin J, Chiarion-Sileni V, Gonzalez R, et al.: Combined nivolumab and ipilimumab or monotherapy in untreated melanoma. N Engl J Med. 2015, 373:23-34. 10.1056/NEJMoa1504030

5. Vanpouille-Box C, Formenti SC, Demaria S: Toward precision radiotherapy for use with immune checkpoint blockers. Clin Cancer Res. 2018, 24:259-265. 10.1158/1078-0432.CCR-16-0037

6. Sharabi AB, Lim M, DeWeese TL, Drake CG: Radiation and checkpoint blockade immunotherapy: radiosensitisation and potential mechanisms of synergy. Lancet Oncol. 2015, 16:e498-e509. 10.1016/\$14702045(15)00007-8

7. Kwon ED, Drake CG, Scher HI, et al.: Ipilimumab versus placebo after radiotherapy in patients with metastatic castration-resistant prostate cancer that had progressed after docetaxel chemotherapy (CA184043): a multicentre, randomised, double-blind, phase 3 trial. Lancet Oncol. 2014, 15:700-712. 10.1016/S1470-2045(14)70189-5

8. Chandra RA, Wilhite TJ, Balboni TA, et al.: A systematic evaluation of abscopal responses following radiotherapy in patients with metastatic melanoma treated with ipilimumab. Oncoimmunology. 2015, 4:e1046028. 10.1080/2162402X.2015.1046028

9. Theurich S, Rothschild SI, Hoffmann M, et al.: Local tumor treatment in combination with systemic ipilimumab immunotherapy prolongs overall survival in patients with advanced malignant melanoma. Cancer Immunol Res. 2016, 4:744-754. 10.1158/2326-6066.CIR-15-0156

10. Antonia SJ, Villegas A, Daniel D, et al.: Durvalumab after chemoradiotherapy in stage III non-small-cell lung cancer. N Engl J Med. 2017, 377:1919-1929. 10.1056/NEJMoa1709937

11. Antonia SJ, Villegas A, Daniel D, et al.: Overall survival with durvalumab after chemoradiotherapy in stage III NSCLC. N Engl J Med. 2018, 379:2342-2350. 10.1056/NEJMoa1809697

12. Gabani P, Robinson CG, Ansstas G, Johanns TM, Huang J: Use of extracranial radiation therapy in metastatic melanoma patients receiving immunotherapy. Radiother Oncol. 2018, 127:310-317. 10.1016/j.radonc.2018.02.022

13. Gabani P, Fischer-Valuck BW, Johanns TM, et al.: Stereotactic radiosurgery and immunotherapy in melanoma brain metastases: patterns of care and treatment outcomes. Radiother Oncol. 2018, 128:266-273. 10.1016/j.radonc.2018.06.017

14. Bilimoria KY, Stewart AK, Winchester DP, Ko CY: The National Cancer Data Base: a powerful initiative to improve cancer care in the United States. Ann Surg Oncol. 2008, 15:683-690. 10.1245/s10434-007-9747-3

15. Winchester DP, Stewart AK, Bura C, Jones RS: The National Cancer Data Base: a clinical surveillance and quality improvement tool. J Surg Oncol. 2004, 85:1-3. 10.1002/jso.10320

16. Boffa DJ, Rosen JE, Mallin K, et al.: Using the National Cancer Database for outcomes research: a review . JAMA Oncol. 2017, 3:1722-1728. 10.1001/jamaoncol.2016.6905

17. Wang SJ, Haffty B: Radiotherapy as a new player in immuno-oncology . Cancers (Basel). 2018, 10:515. 10.3390/cancers 10120515

18. Postow MA, Callahan MK, Barker CA, et al.: Immunologic correlates of the abscopal effect in a patient with melanoma. N Engl J Med. 2012, 366:925-931. 10.1056/NEJMoa1112824

19. Seung SK, Curti BD, Crittenden M, et al.: Phase 1 study of stereotactic body radiotherapy and interleukin-2-tumor and immunological responses. Sci Transl Med. 2012, 4:137-174. 10.1126/scitranslmed.3003649

20. Twyman-Saint Victor C, Rech AJ, Maity A, et al.: Radiation and dual checkpoint blockade activate nonredundant immune mechanisms in cancer. Nature. 2015, 520:373-377. 10.1038/nature14292

21. Chicas-Sett R, Morales-Orue I, Rodriguez-Abreu D, Lara-Jimenez P: Combining radiotherapy and ipilimumab induces clinically relevant radiation-induced abscopal effects in metastatic melanoma patients: a systematic review. Clin Transl Radiat Oncol. 2018, 9:5-11. 10.1016/j.ctro.2017.12.004

22. Wild AT, Herman JM, Dholakia AS, et al.: Lymphocyte-sparing effect of stereotactic body radiation therapy in patients with unresectable pancreatic cancer. Int J Radiat Oncol Biol Phys. 2016, 94:571-579. 10.1016/j.ijrobp.2015.11.026

23. Lee Y, Auh SL, Wang Y, et al.: Therapeutic effects of ablative radiation on local tumor require CD8+ T cells: changing strategies for cancer treatment. Blood. 2009, 114:589-595. 10.1182/blood-2009-02-206870

24. Yovino S, Kleinberg L, Grossman SA, Narayanan M, Ford E: The etiology of treatment-related lymphopenia in patients with malignant gliomas: modeling radiation dose to circulating lymphocytes explains clinical observations and suggests methods of modifying the impact of radiation on immune cells. Cancer Invest. 


\section{Cureus}

2013, 31:140-144. 10.3109/07357907.2012.762780

25. Brooks ED, Schoenhals JE, Tang C, Micevic G, Gomez DR, Chang JY, Welsh JW: Stereotactic ablative radiation therapy combined with immunotherapy for solid tumors. Cancer J. 2016, 22:257-266.

10.1097/PPO.0000000000000210

26. Chajon E, Castelli J, Marsiglia H, De Crevoisier R: The synergistic effect of radiotherapy and immunotherapy: a promising but not simple partnership. Crit Rev Oncol Hematol. 2017, 111:124-132. 10.1016/j.critrevonc.2017.01.017

27. Prins RM, Vo DD, Khan-Farooqi H, et al.: NK and CD4 cells collaborate to protect against melanoma tumor formation in the brain. J Immunol. 2006, 177:8448-8455. 10.4049/jimmunol.177.12.8448

28. Wilson EH, Weninger W, Hunter CA: Trafficking of immune cells in the central nervous system . J Clin Invest. 2010, 120:1368-1379. 10.1172/JCI41911

29. Margolin K, Ernstoff MS, Hamid O, et al.: Ipilimumab in patients with melanoma and brain metastases: an open-label, phase 2 trial. Lancet Oncol. 2012, 13:459-465. 10.1016/S1470-2045(12)70090-6

30. Bentzen SM, Overgaard J, Thames HD, Overgaard M, Hansen PV, von der Maase H, Meder J: Clinical radiobiology of malignant melanoma. Radiother Oncol. 1989, 16:169-182. 10.1016/0167-8140(89)90017-0 